\title{
PLAN DE MANEJO DEL ZÚNGARO TIGRINUS Brachyplatystoma tigrinum (Britski, 1981) EN LA AMAZONÍA PERUANA
}

\author{
Fernando ALCÁNTARA ${ }^{1}$, Fred CHU-KOO ${ }^{1}$, Luciano RODRÍGUEZ ${ }^{1}$, Carlos CHÁVEZ ${ }^{1}$, Salvador \\ TELLO ${ }^{1}$, Aurea GARCÍA ${ }^{1}$, Germán MURRIETA ${ }^{2}$, Jorge MORI ${ }^{2}$, Jesús NUÑEZ ${ }^{3}$, Carmen GARCÍA- \\ DÁVILA ${ }^{1}$, Fabrice DUPONCHELLE ${ }^{3}$
}

1 Instituto de Investigaciones de la Amazonía Peruana, IIAP. Programa para el Uso y Conservación del Agua y sus Recursos (AQUAREC), Carretera Iquitos-Nauta, Km 4.5. Iquitos, Perú, E.mail : fab_001@hotmail.com

2 Universidad Nacional de la Amazonía Peruana - UNAP. Escuela de Post Grado. Cátedra CONCYTEC. Maestría en Acuicultura. Iquitos, Perú.

3 Institut de Recherche pour le Développement-IRD. 175 Caviar, BP 5095, 34196 Montpellier cedex 05, France.

\section{RESUMEN}

El zúngaro tigrinus Brachyplatystoma tigrinum (Pimelodidae) es un pez que, en su medio natural, alcanza $85 \mathrm{~cm}$ de longitud y 5,200 g de peso, habita la corriente del río Amazonas y es capturado por pescadores de peces ornamentales al estado de alevino y, ocasionalmente, por pescadores de peces de consumo, en el tramo comprendido entre Tamishiyacu y la Refinería Luis F. Díaz; aguas arriba y aguas abajo de Iquitos, respectivamente. B. tigrinum ocupa el tercer lugar de exportación, situándose en orden de importancia después de la arahuana Osteoglossum bicirrhosum y el peje torre Phractocephlaus hemiliopterus en la pesquería de peces ornamentales con base en Iquitos, Perú. Por esta razón, un sector importante de la población se dedica a la pesca, acopio, cría y comercialización de esta especie, impactando la reposición de sus poblaciones naturales. Como consecuencia la Dirección Regional de Loreto del Ministerio de la Producción, mediante planes de manejo propuestos por la Asociación de Pescadores de Peces Ornamentales de Loreto, Iquitos, APPOLI, establece cuotas anuales de captura de alevinos. En este artículo se propone un plan de manejo del zúngaro tigrinus $B$. tigrinum que considera estrategias orientadas a mejorar el conocimiento y la administración del recurso, reduciendo la cuota anual de pesca de 6,000 a 5,000 alevinos.

PALABRAS CLAVE: Zúngaro tigrinus Brachyplatystoma tigrinum, plan de manejo, cuota anual de captura.

\section{MANAGEMENT PLAN FOR ZÚNGARO TIGRINUS Brachyplatystoma tigrinum (Britski, 1981) IN THE PERUVIAN AMAZON}

\begin{abstract}
The catfish zúngaro tigrinus Brachyplatystoma tigrinum (Pimelodidae) reaches in the wild $85 \mathrm{~cm}$ in length and $5.200 \mathrm{~g}$ in weight, inhabits the main channel of the Amazon River . It is captured by fishermen as a fry for the ornamental trade and occasionally as an adult for human consumption in the section between Tamishiyacu and Luis F. Refinery Diaz upstream and downstream from Iquitos, respectively. B. tigrinum is the third most important exported species after the arahuana Osteoglossum bicirrhosum and Peje torre Phractocephlaus hemiliopterus in the ornamental fishery based in Iquitos, Peru. For this reason, an important sector of the population engages in fishing, gathering, breeding and marketing this species, greatly impacting their natural populations. As a result the Regional Direction of Loreto of the Ministry of Production, following management plans proposed by the Fishermen's Association of Ornamental Fish Loreto, Iquitos, APPOLI, sets annual catch quota of fry. This article proposes a management plan for $B$. tigrinum aiming at improving the knowledge and management of this important resource, and proposes to reduce the annual fry catch 6.000 to 5.000 .
\end{abstract}

KEYWORDS: Zúngaro tigrinus Brachyplatystoma tigrinum, management plan, annnual catch. 


\section{INTRODUCCIÓN}

El zúngaro tigrinus Brachyplatystoma tigrinum (Britski, 1981), es un pez que pertenece al grupo de los grandes bagres o Pimelodidos que, los pescadores de peces ornamentales $y$, eventualmente, los pescadores de peces de consumo, capturan en la corriente del río Amazonas con aparejos a la deriva, guiados por una o dos embarcaciones.

Recientemente fueron realizadas observaciones sobre biología, las épocas de pesca, aparejos de pesca, acopio, transporte, manejo en las instalaciones comerciales (Chávez et al., 2008; Alcántara et al., 2010); así como, sobre la relación entre la longitud y el peso y el factor de condición (Alcántara et al., 2008a); parasitismo por Argulus pestifer (Alcántara et al., 2008b); alimentación y cría en diferentes condiciones de alimentación, sistemas de flujo continuo y cerrado y cultivo en diferente calidad de agua (Murrieta, 2009); así como, sobre genética de la especie (Mori, 2010).

Al estado adulto no es registrada su presencia en los desembarques de la flota comercial y al estado de alevino, su captura anual, está regulada por planes de manejo propuestos por la Asociación de Pescadores de Peces Ornamentales de Loreto Iquitos - APPOLI, aprobados por la Dirección Regional de Loreto del Ministerio de la Producción (APPOLI, 2009).

En este contexto, se propone este plan alternativo de manejo del zúngaro tigrinus Brachyplatystoma tigrinum en Iquitos, Loreto, para el establecimiento de nuevas estrategias de administración del recurso y de cuotas de captura anual.

\section{PROPUESTA DEL PROGRAMA DE MANEJO DEL ZÚNGARO TIGRINUS EN LA AMAZONÍA PERUANA}

La Resolución Ministerial $N^{\circ}$ 147-2001-PE que aprueba el Reglamento de Ordenamiento Pesquero de la Amazonía Peruana establece la estructura para la formulación y ejecución de un Programa de Manejo Pesquero (MAPE). En este sentido, esta propuesta se ciñe a la norma indicada.

\section{LA ESPECIE Y CARACTERIZACIÓN DEL ESPACIO ECOLÓGICO}

Estos aspectos son reportados por Chávez et al. (2009), Alcántara et al. (2008a), Alcántara et al. (2008b) y Alcántara et al. (2010).

\section{JUSTIFICACIÓN DEL MAPE}

El zúngaro tigrinus Brachyplatystoma tigrinum (Britski, 1981) es un pez de porte grande que alcanza hasta $85 \mathrm{~cm}$ y $5200 \mathrm{~g}$ que, vive en el curso de la corriente de los grandes ríos, como el Amazonas y el Marañón, en los que se reproduce estacionalmente, entre octubre y abril que, corresponde al final de la vaciante y comienzos de la creciente del ambiente acuático.

El zúngaro tigrinus tiene un hábitat de difícil acceso a los aparejos de pesca tradicionales de uso corriente en la captura de peces en la Amazonía peruana; por esta razón su captura es escasa y no es frecuente su observación en los desembarques de la flota comercial de peces de consumo que opera en el mismo tipo de ambiente para capturar las especies del grupo de los grandes bagres, como el dorado Brachyplatystoma rousseauxii, el saltón Brachyplatystoma filamentosum, el peje torre Phractocephalus hemiliopterus, la doncella Pseudoplatystoma punctifer, el tigre zúngaro Pseudoplatystoma tigrinum entre otras.

Sin embargo, pescadores de peces ornamentales, desde hace un poco menos de una década aprendieron a capturar al zúngaro tigrinus al estado de alevino, con tallas de 2 a $5 \mathrm{~cm}$, en la corriente de pequeños brazos del río Amazonas, en áreas en las que, en vaciante, se cultiva arroz (Figuras 1 y 2 ). La disponibilidad y la captura son bajas y en una faena de pesca pueden ser capturados de uno a cinco o seis ejemplares y excepcionalmente, un número mayor.

Los pescadores y luego los acopiadores, entonces, inician un proceso de cría de estos zúngaros hasta que alcanzan tallas de 15 a $18 \mathrm{~cm}$ para su venta a los exportadores de peces ornamentales, a precios que pueden llegar a cincuenta nuevos soles (U. S. \$ 15.00) en el mercado local. Por tanto, el zúngaro tigrinus constituye una especie importante en la pesquería de peces ornamentales, en Iquitos, por su valor económico que puede llegar a los \$60,000 anuales (Moreau \& Cooms, 2007) y, porque constituye una fuente de empleo para numerosas personas que participan en la pesca, transporte, cría y manejo de la especie.

En concordancia con lo expuesto, la Dirección Regional de Loreto del Ministerio de la Producción PRODUCE, desde el 2003 viene estableciendo cuotas de pesca (Tabla 1), basadas en la propuesta de Planes de Manejo Pesquero, para asegurar el uso sostenido de la especie, en Iquitos. Este plan de manejo, constituye una propuesta alternativa a los planes propuestos por la Asociación de Pescadores de Loreto, Iquitos APPOLI. 


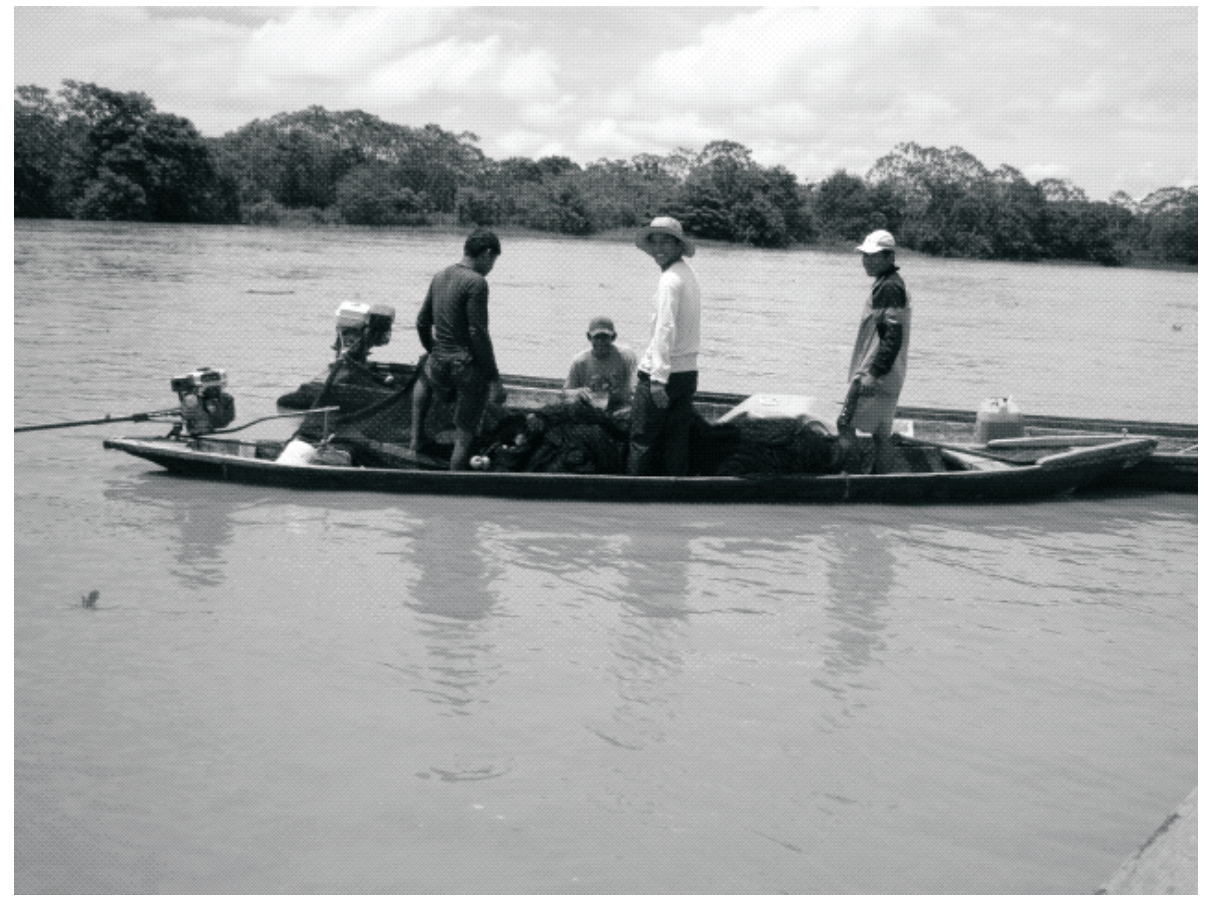

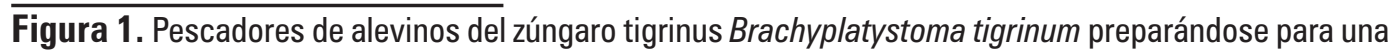
faena de pesca en el río Amazonas.

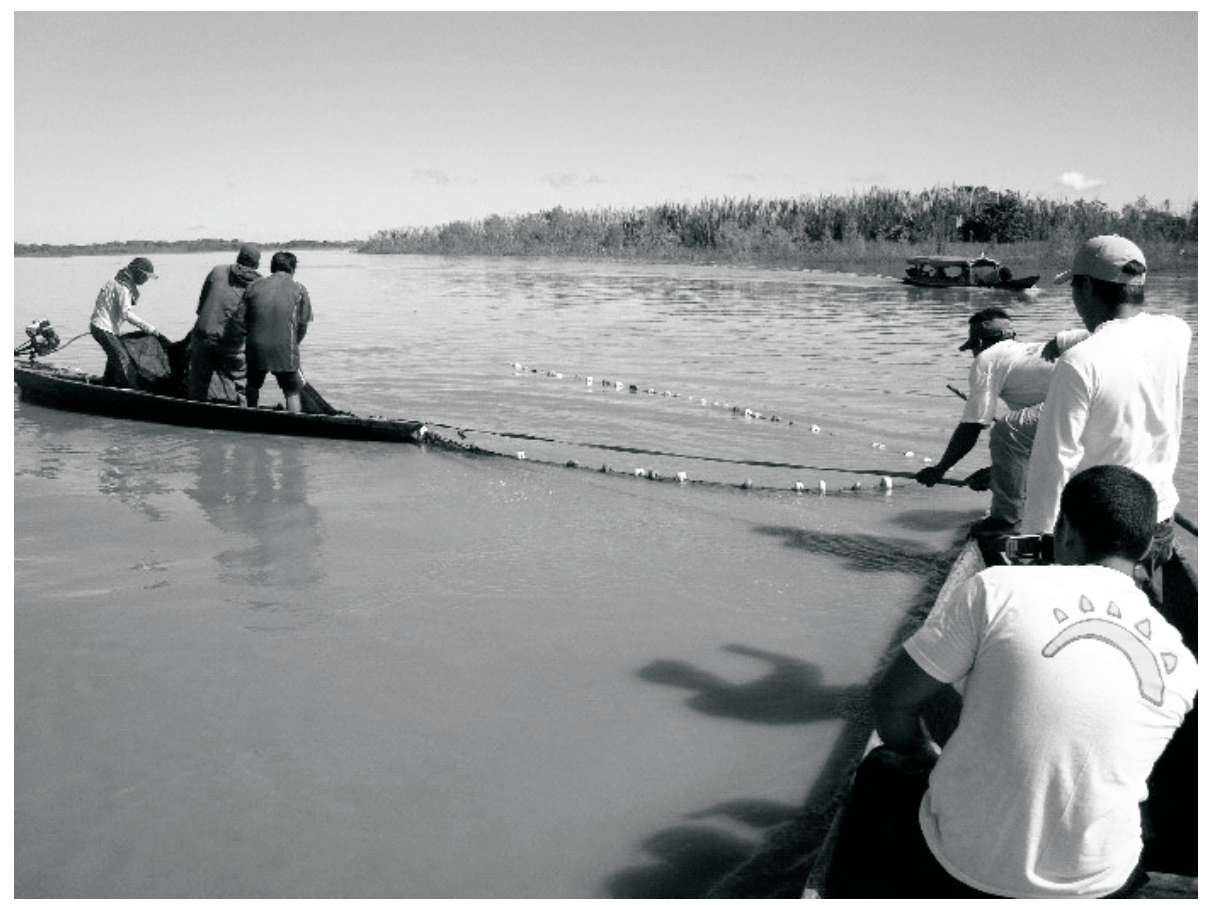

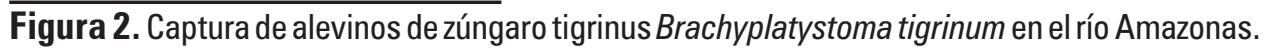




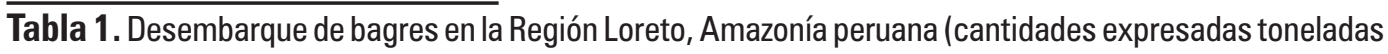
métricas).

\begin{tabular}{l|l|c|c|c|c|c|c}
\hline $\mathbf{N}^{\mathbf{0}}$ & ESPECIE & $\mathbf{2 0 0 3}$ & $\mathbf{2 0 0 4}$ & $\mathbf{2 0 0 5}$ & $\mathbf{2 0 0 6}$ & $\mathbf{2 0 0 7}$ & $\mathbf{2 0 0 7}$ \\
\hline & Achacubo & 8.1 & 10.3 & 14,10 & 19.2 & 29.35 & 0.97 \\
\hline Alianza & 4.18 & 4.78 & 11.05 & 13.72 & 15.21 & 0.50 \\
\hline Achara & 3.75 & 5.22 & 10.15 & 14.76 & 19.49 & 0.65 \\
\hline Bagre & 112.56 & 56.96 & 74.09 & 69.45 & 95.53 & 3.17 \\
\hline Cachorro & 0 & 0 & 0 & 1.78 & 4.1 & 0.14 \\
\hline Cunchimama & 108.61 & 55.69 & 50.52 & 78.35 & 97.03 & 3.22 \\
\hline Doncella & 2252.46 & 205.44 & 162 & 278.72 & 304.35 & 10.09 \\
\hline Dorado & 78.76 & 54.74 & 53.86 & 58.63 & 66.5 & 2.21 \\
\hline Manitoa & 65.75 & 38.99 & 102.17 & 113.23 & 119.26 & 3.96 \\
\hline Torre & 61.35 & 36.31 & 47.61 & 52.33 & 57.89 & 1.92 \\
\hline Saltón & 49.12 & 45.38 & 40.86 & 39.53 & 47.73 & 1.58 \\
\hline Tigre & 98.29 & 44.04 & 46.19 & 51.34 & 64.38 & 2.14 \\
\hline Zorro & 0 & 0 & 0 & 0 & 0 & 0.00 \\
\hline Tabla barba & 2.01 & 3.28 & 8.26 & 7.65 & 9.89 & 0.33 \\
\hline Toa & 41.25 & 2.1 & 5.17 & 4.76 & 3.04 & 0.10 \\
\hline Mota & 0 & 53.03 & 52.72 & 61.3 & 74.5 & 2.47 \\
\hline Total & 4889.19 & 2620.26 & 2669.65 & 2870.75 & 3015.25 & 33.45 \\
\hline
\end{tabular}

Nota: El desembarque registrado en el 2007 se convirtió a cifras relativas para verificar el porcentaje de pesca del zúngaro tigrinus y del zúngaro alianza en relación a los otros bagres.

Tabla 2. Cuotas de captura de zúngaro tigrinus Brachyplatystoma tigrinum y zúngaro alianza Brachyplatystoma juruensis, por período de pesca en el río Amazonas. APPOLI. 2009.

\begin{tabular}{|c|c|c|c|c|c|c|c|c|}
\hline \multirow{2}{*}{ ESPECIE } & \multicolumn{2}{|c|}{ 2003-2007 } & \multicolumn{2}{|c|}{ 2007-2008 } & \multicolumn{2}{|c|}{ 2008-2009 } & \multicolumn{2}{|c|}{ 2009-2010 } \\
\hline & CUOTA & DEVOL. & CUOTA & DEVOL. & CUOTA & DEVOL. & CUOTA & DEVOL. \\
\hline Zúngaro Tigrinus & 10,575 & & 3,500 & 350 & 3,500 & & 6,000 & 660 \\
\hline Zúngaro alianza & 20,384 & & 7,000 & 700 & 3,700 & & 6,000 & 660 \\
\hline TOTAL & 30,959 & & 10,500 & 1,050 & 7,200 & & 12,000 & 1,320 \\
\hline
\end{tabular}

Nota.- En algunos casos se indica la cifra de devolución a sus zonas naturales, como una acción de repoblamiento, luego de un período de cría a cargo de los pescadores y acopiadores de APPOLI 


\section{RESULTADOS ESPERADOS}

- Mejor conocimiento de la especie, en lo referente a su distribución en la región amazónica del país, así como sobre sus hábitos alimenticios, su fecundidad, sus áreas de desove, épocas de reproducción, entre otros.

- Mejor conocimiento sobre las áreas de pesca.

- Establecimiento de cuotas de captura teniendo como base un mejor conocimiento de la explotación del recurso.

- Establecimiento de protocolos de manejo de alevinos en condiciones controladas.

- Uso sostenido del recurso.

\section{DERECHOS Y OBLIGACIONES}

\section{DERECHOS}

a. La Asociación de Pescadores de Peces Ornamentales de Loreto, Iquitos APPOLI es la beneficiaria de las cuotas de pesca que determine la Dirección Regional de Loreto del Ministerio de la Producción.

b. Las cuotas de captura serán establecidas periódicamente, a criterio de la Dirección Regional de Loreto del Ministerio de la Producción, en función de los avances en las investigaciones que realice el Instituto de Investigaciones de la Amazonía Peruana IIAP.

c. La Dirección Regional de Loreto del Ministerio de la Producción, como una alternativa de promover la asociatividad de los pescadores y acopiadores del zúngaro tigrinus, solo concederá permiso de pesca a APPOLI, absteniéndose de otorgar permiso de pesca, en forma individual para la captura del zúngaro tigrinus, sea que se trate al estado de alevino, o al estado de adulto.

d. APPOLI determinará, en asamblea de socios, las cuotas individuales por equipo $\mathrm{y}$, por temporada de pesca.

\section{OBLIGACIONES}

a. APPOLI se obliga a llevar un Registro de socios que, tendrá el carácter de Declaración Jurada y servirá como requisito para la renovación del permiso de pesca para cada temporada.

b. APPOLI se obliga a llevar un Registro de las áreas de pesca, temporadas de pesca, aparejos de pesca, accesorios y procedimientos utilizados en el manejo del zúngaro tigrinus.

c. APPOLI se obliga a llevar un registro de captura indicando la fecha, el lugar de captura, el número de tigrinus capturados por faena de pesca, anotando, además, el tipo de embarcación o embarcaciones utilizadas, uso de redes, motores, caballaje, pescadores por embarcación.

d. APPOLI se obliga a tener actualizados sus libros de registro de información y disponibles para la supervisión que será ejercida por la Dirección Regional de PRODUCE.

\section{CONOCIMIENTO PREVIO DEL RECURSO Y PLAN DE MANEJO PROPUESTO POR APPOLI (Resumen)}

La Asociación de Pescadores de Peces Ornamentales de Loreto, Iquitos, APPOLI, captura el zúngaro tigrinus y el zúngaro alianza (Pimelodidae) en el río Amazonas, principalmente, en el tramo comprendido entre Panguana, Primera y Segunda Zona y el caserío Santa Rosa, frente a la base del SIMAI. Sin embargo, los pescadores de peces de consumo especializados en la captura de grandes bagres capturan también estas especies en la corriente del río, en especial en la zona de costa brava, en el sector de Tamishiyacu.

En el PROMAPE que propone la APPOLI se observa un cuadro referente al desembarque de bagres "en la región Loreto" correspondiente al periodo 2003 2007 (Tabla 1), en el que se aprecia la incidencia en la captura del zúngaro alianza. Por la información que contiene esta tabla, se presenta como antecedente.

El PROMAPE del zúngaro tigrinus y del zúngaro alianza, en la Amazonía peruana está en aplicación desde el año 2003, en que la DIREPRO asigna cuotas de captura a APPOLI (Tabla 2).

En los registros de desembarque de bagres en la Región Loreto de la DIREPRO (Tabla 1), se observa que la doncella ocupa el primer lugar, seguida de manitoa, cunchimama, bagre y otras y que, el zúngaro alianza ocupa apenas un $0.5 \%$ del desembarque total equivalente a 3,015.25 toneladas. En este sentido, cabe indicar que, el zúngaro tigrinus no aparece en el desembarque de bagres en la región Loreto, salvo que haya sido reportado como zúngaro alianza. No obstante, cabe indicar que, durante la ejecución del sub proyecto "Reproducción inducida, manejo de alevinos y uso sostenido del zúngaro tigrinus Brachyplatystoma tigrinum (Britski, 1981) en la Amazonía peruana" se efectuaron muestreos de los desembarques de peces de consumo en los diferentes puertos de Iquitos, sin resultados de detección de especimenes.

Por otra parte, cabe indicar que, aun cuando se nota un incremento en el desembarque de zúngaro alianza de 4 a $15 \mathrm{t}$, en el periodo en consideración, puede afirmarse que, la captura de esta(s) especie(s) es poco frecuente. Asimismo, se debe anotar que, globalmente, se observa que el desembarque total de grandes bagres, 
durante los últimos cinco años es más o menos equivalente, con excepción del año 2003 en que se obtuvo una captura mayor al promedio.

La captura del zúngaro tigrinus efectuada en el río Marañón, cerca del Pongo de Manseriche (Sánchez, 2008) indica que la especie tiene amplia distribución pero su captura es poco frecuente. Aquí se debe tener en cuenta que, las referencias de los pescadores de APPOLI indican que el zúngaro tigrinus vive, a través de su desarrollo, en las palizadas de la corriente del río, donde no son accesibles a los aparejos de pesca tradicionales.

El PROMAPE que propone APPOLI está focalizado a un corto tramo del río Amazonas, comprendido entre Tamishiyacu y Santa Rosa y no considera información sobre la disponibilidad del zúngaro tigrinus y del zúngaro alianza, en otras áreas de pesca.

En la tabla 2, referente al establecimiento de cuotas de captura del zúngaro tigrinus desde 2003 a la fecha, se observa que, además del establecimiento de las cuotas de captura, se indica el establecimiento de una cuota de devolución o repoblamiento del ambiente natural que, se realiza luego de un período de cría de los alevinos del zúngaro tigrinus que realizan los pescadores o acopiadores, como mecanismo de compensación de la extracción y, con la finalidad de asegurar el uso sostenido del recurso. Esta práctica, sin embargo, supone la readaptación al medio natural de una especie capturada con tallas de 2 a $5 \mathrm{~cm}$, criada con oferta sistemática de alimento, sin competidores ni predadores, hasta que alcanza longitudes de 18 a $20 \mathrm{~cm}$ para su liberación al medio natural o repoblamiento. Se desconoce la validez de este procedimiento y la consecuente readaptación de la especie, al medio natural, luego de ser adaptada al cautiverio. Por tanto, sería preferible descontar la cifra de devolución de la cuota de captura a asignarse por periodo.

\section{INVESTIGACIÓN Y SEGUIMIENTO}

El Instituto de Investigaciones de la Amazonía Peruana IIAP, en cumplimiento de sus funciones de investigación y evaluación de los recursos naturales renovables de la región amazónica se encargará de la ejecución de las investigaciones necesarias, del recurso, en orden a determinar su estado situacional y promover su uso sostenido para el bienestar de la población.

En concordancia con lo expuesto, el IIAP, en coordinación con la DIREPRO Loreto podrán ejercer la supervisión de las acciones de captura, acopio y manejo del zúngaro tigrinus en la Región Loreto, fiscalizando, además, los registros que llevará APPOLI.

\section{TÉCNICAS DE MANEJO}

La propuesta de este Plan de Manejo del zúngaro tigrinus, alternativo al PROMAPE que viene proponiendo APPOLI a PRODUCE, con la finalidad de establecimiento de las cuotas de captura para la temporada, se basa en entrevistas a pescadores, acopiadores, comercializadores, pescas experimentales y revisión de la información que proporciona APPOLI en su propuesta de plan de manejo para el período $2009-2010$.

Las técnicas de manejo del zúngaro tigrinus $B$. tigrinum (Bristki, 1981) en la Amazonía peruana, en nuestra opinión, deben centrar el esfuerzo en los tópicos que se indica a continuación.

\section{A. MEJORAMIENTO DEL CONOCIMIENTO SOBRE LA BIOLOGIADE LAESPECIE}

En nuestra opinión, el plan de manejo del tigrinus debe tener como base el mejoramiento del conocimiento de la biología de la especie, en especial, en lo referente a sus épocas de reproducción, lugares de desove y desarrollo larval y de alevinaje. Si bien los alevinos son capturados en el curso de la corriente del río Amazonas, se debe agotar esfuerzos por conocer con precisión las épocas de maduración gonadal, así como, los lugares de puesta y desarrollo larval. En este sentido, se propone que la Dirección Regional de Loreto, del Ministerio de la Producción PRODUCE, establezca los mecanismos necesarios para acopiar información biológica (talla, peso, sexo, grado de madurez según la escala de maduración sexual proporcionada por el IIAP que, incluya el registro fotográfico de cada gónada), lugares de captura del tigrinus, duración de la temporada de pesca y las características del ambiente. Esta información puede ser acopiada por los integrantes de APPOLI durante sus faenas de pesca.

Los datos biológicos colectados de la especie en consideración, serán analizados por el IIAP.

\section{B. EFECTUAR ESTUDIOS DE REPRODUCCIÓN DEL TIGRINUS EN AMBIENTE CONTROLADO}

Se debe propiciar la continuación de los estudios de reproducción para lograr una tecnología de producción de crías, segura y, en concordancia con la demanda del mercado. De esta forma, el abastecimiento de alevinos procedentes de la reproducción inducida en condiciones controladas puede contribuir a disminuir la presión de pesca de la especie, mejorando las posibilidades de su uso sostenido. 


\section{ESTABLECIMIENTO DE CUOTAS ANUALES DE CAPTURA}

Considerando que la pesca del tigrinus en la amazonía peruana y sobre todo, en Iquitos, juega un rol socio económico importante que, proporciona empleo e ingresos a un sector importante de la población, se propone el establecimiento de una cuota anual de captura de 5,000 alevinos. En esta cuota, no se considera el porcentaje de devolución previsto en los planes de manejo, previos propuestos por APPOLI. Es decir, se propone una cuota fija, en la cual, la captura total sería criada y vendida por los integrantes de APPOLI. Dicho de otro modo, la cuota de reposición simplemente se dejaría de pescar, con fines de propiciar el uso sostenido del recurso.

\section{EFECTUAR EL MONITOREO DE LA CAPTURA $Y$ COMERCIALIZACION DEL TIGRINUS EN IQUITOS}

Precautoriamente, la Dirección Regional de Iquitos del Ministerio de la Producción debe supervisar el cumplimiento de las cuotas de captura establecidas, monitoreando las acciones de APPOLI y la comercialización de los acuarios comerciales de Iquitos.

\section{E. ELABORACION DE UNA BASE DE DATOS DE CAPTURA Y COMERCIALIZACION}

Complementariamente a las acciones de supervisión del cumplimiento de las cuotas de captura, la Dirección Regional de Iquitos del Ministerio de la Producción debe llevar una base de datos de la captura y comercialización del tigrinus la que, luego del análisis, correspondiente, permitirá determinar las tendencias del estado del recurso, en orden a asegurar su uso sostenido.

\section{F. ELABORACION DE UNA BASE DE DATOS SOBRE ASPECTOS SOCIO ECONOMICOS DE LA PESCA Y COMERCIALIZACION DEL TIGRINUS}

Igualmente, la Dirección Regional de Iquitos del Ministerio de la Producción debe llevar una base de datos de los aspectos socio económicos relacionados con la captura y comercialización del tigrinus, en orden a determinar su impacto socio económico.

\section{G. CRIA DE TIGRINUS EN CONDICIONES CONTROLADAS}

El IIAP debe continuar la cría del zúngaro tigrinus con la finalidad de lograr la generación de tecnología de producción de crías de esta especie, en ambiente controlado. Por eso y a fin de lograr el desarrollo gonadal de los progenitores en condiciones de cultivo, se deberán adaptar sus condiciones de mantenimiento a las condiciones de vida de la especie en ambientes naturales, usando un circuito cerrado con flujo de agua, temperatura y fotoperíodo controlados, para lo cual, se deben orientar esfuerzos para conseguir el financiamiento correspondiente.

\section{H. UTILIZACIÓN DE LOS RECURSOS}

La utilización actual del zúngaro tigrinus se realiza, principalmente, como pez ornamental, al estado de alevino, con tallas de 15 a $20 \mathrm{~cm}$ en que son exportados por los comercializadores mayoristas de peces ornamentales de Iquitos. Es decir, la utilización del recurso ocurre luego de un período de cría ya que, los pescadores lo capturan, preferentemente, con tallas de 2 a $5 \mathrm{~cm}$ y luego lo crían, en condiciones controladas, en acuarios, con alimentos naturales diversos (peces forraje) hasta que los peces alcanzan las tallas de comercialización nacional y externa.

Debido a su escasa incidencia en la pesquería de peces de consumo, esta especie no es utilizada en el consumo humano directo.

\section{VIGILANCIAY CONTROL}

La DIREPRO Loreto supervisa, periódicamente, el cumplimiento de las regulaciones, no obstante, se recomienda llevar un buen registro de la salida de los peces hacia los mercados nacional y externo. Esta información debe ser contrastada con los registros de desembarque de APPOLI para tener un control de la extracción.

\section{J. EVALUACIÓN DEL MAPE}

La DIREPRO Loreto debe organizar, anualmente, un taller de evaluación del MAPE del tigrinus en el que se debe considerar los aspectos siguientes:

- Áreas de pesca

- Aparejos de pesca

- Embarcaciones

- Equipamiento con motores fuera de borda, indicando el tipo y potencia

- Disponibilidad del recurso: Captura/ lance, Captura/faena de pesca

- Tallas de captura

- Transporte: Métodos

- Métodos de manejo en instalaciones de cría: Calidad de agua, alimentos, frecuencia de oferta, horario de oferta, etc

- Prevención y control de patógenos

- Tallas de comercialización 
- Precios de comercialización

- Acuarios de destino

- Cantidad vendida

- Mortalidad en acuario

- Pescadores que operaron en la temporada de pesca

- Niveles de ingresos

- Cumplimiento de las cuotas de captura establecidas en el MAPE

- Análisis del MAPE: Que se cumplió? Que es lo funcional?

- Propuestas de adecuación del MAPE

\section{K. AJUSTES DE PRODUCCIÓN}

Luego del taller de evaluación del MAPE en vigencia y de las tendencias del recurso, la DIREPRO Loreto, en coordinación con el IIAP, harán los ajustes de producción para la temporada de pesca siguiente.

\section{OTRAS MEDIDAS DE CONSERVACIÓN DEL RECURSO}

En función de la demanda, principalmente del mercado externo y asumiendo que la especie se encuentra presente en el río Ucayali, IIAP-PRODUCE harán la transferencia de conocimiento y tecnología a la DIREPRO Ucayali.

\section{AGRADECIMIENTOS}

Expresamos nuestro agradecimiento al Sr. Sixto Nautino, integrante de APPOLI y su equipo de pescadores, por habernos proporcionado información sobre captura y manejo de alevinos del zúngaro tigrinus $y$, por habernos permitido participar en sus faenas de pesca de alevinos en el río Amazonas. Igualmente queremos dejar constancia de nuestro agradecimiento al Sr. Rigoberto Tangoa, pescador de grandes bagres de la localidad de Tamishiyacu por habernos permitido participar en sus faenas de pesca en el río Amazonas.

Asimismo, expresamos nuestro reconocimiento a INCAGRO por el financiamiento del sub proyecto de investigación "Reproducción inducida, manejo de alevinos y uso sostenido del zúngaro tigrinus Brachyplatystoma tigrinum (Britski, 1981) en la Amazonía peruana", durante el periodo 2008 -2010.

\section{BIBLIOGRAFÍAS CITADAS}

Alcántara, F., Chu-Koo, F., Rodríguez, L., Chávez, C., Tello, S. \& Nuñez, J. 2008. Relación longitud peso y el factor de condición del tigrinus Brachyplatystoma tigrinum del río Amazonas. Loreto. Perú. Folia Amazónica No17 (1-2):23-28
Alcántara, F., Chu-Koo, F., Rodríguez, L., Chávez, C., Bernuy, A., Barbarán, T., Tello, S. \& Núñez, J. 2008. Primer reporte de parasitismo de Brachyplatystoma tigrinum por Argulus pestifer en acuicultura. Folia Amazónica Vol. 17 (1-2) :99-102

Alcántara, F., Chu-Koo, F., Rodríguez, L., Chávez, C., Bernuy, A., Barbarán, A., Tello, S. Murrieta, G., Nuñez, J. 2010. Notas sobre biología y pesca del zúngaro tigrinus Brachyplatystoma tigrinum en el río Amazonas. Instituto de Investigaciones de la Amazonia Peruana. Universidad Nacional de la Amazonía Peruana. Institut de Recherche pour le Developpement-IRD. 18 p. Por publicar.

Asociación de pescadores de peces ornamentales de Loreto, Iquitos, APPOLI. 2009. Programa de Manejo Pesquero de Alevinos de tigrinus Merodontotus tigrinus y zúngaro alianza Brachyplatystoma juruense. Base legal D. S. No 015-2009-PRODUCE. Iquitos. Perú. 30 p.

Chávez, C, Bernuy, A., Barbarán, T., Murrieta, G., Sánchez, H., Chu-Koo, F., Alcántara, F., Nuñez, J. 2008. Reporte de los conocimientos tradicionales sobre el zúngaro tigrinus Brachyplatystoma tigrinum en la Amazonía peruana. IIAP - IRD. 6 p. Por publicar.

Malta, J.C.O., 1983. Os argulídeos (Crustacea: Branchiura) da Amazônia Brasileira. 4. Aspectos da ecologia de Argulus multicolor Stekhoven, 1937 e A. pestifer Ringuelet, 1948. Acta Amazonica, 13(2):489-496.

Malta, J.C.O., \& Varela, A.M.B. 2000. Argulus chicomendesi sp. n. (Crustacea: Argulidae) Parasita de peixes da Amazonia Brasileira. Acta amazonica 30 (1): 481 - 498.

Moreau, Marie- Annick \& Cooms, O. 2007. Aquarium fish exploitation in western Amazonía: Conservation issues in Peru. Foundation for Environmental Conservation. Environmental Conservation 34 (1): 12-22.

Mori, M. Jorge. 2010. Estudio comparativo de la variabilidad genética y filogenética del zúngaro tigrinus Brachyplatystoma tigrinum (Britski, 1981) y especies relacionadas de la familia Pimelodidae en la Amazonía Peruana. Tesis para optar el título profesional de Biólogo. Universidad Nacional de la Amazonía Peruana. Facultad de Ciencias Biológicas. 55 p.

Murrieta, G. 2009. Manejo de alevinos de zúngaro tigrinus Brachyplatystoma tigrinum (Britski, 1981) (Pisces, Pimelodidae) en condiciones controladas. Tesis para optar el titulo profesional de Biologo. Universidad Nacional de la Amazonia Peruana. $42 \mathrm{p}$ Por publicar.

Sánchez, H. 2008. Reporte sobre la ictiofauna del alto Marañón. IIAP. Informe interno. 12 p. 

